Article

\title{
Synthesis and Biological Evaluation of Unsymmetrical Curcumin Analogues as Tyrosinase Inhibitors
}

\author{
Yongfu Jiang ${ }^{1,2}$, Zhiyun Du ${ }^{1,3, *}$, Guihua Xue ${ }^{1}$, Qian Chen ${ }^{1}$, Yujing Lu ${ }^{1}$, Xi Zheng ${ }^{1}$, \\ Allan H. Conney ${ }^{1}$ and Kun Zhang ${ }^{1, *}$ \\ 1 Institute of Natural Medicine \& Green Chemistry, School of Chemical Engineering and Light Industry, \\ Guangdong University of Technology, Guangzhou 510006, China \\ 2 Department of Chemistry, Zhejiang Normal University, Jinhua 321004, China \\ 3 State Key Laboratory of Natural and Biomimetic Drugs, Peking University, Beijing 100191, China \\ * Authors to whom correspondence should be addressed; E-Mails: zhiyundu@gdut.edu.cn (Z.D.); \\ kzhang@gdut.edu.cn (K.Z.); Tel.: +86-20-3932-2235 (Z.D.).
}

Received: 31 January 2013; in revised form: 14 March 2013 / Accepted: 22 March 2013 /

Published: 3 April 2013

\begin{abstract}
Synthesis and biological evaluation of unsymmetrical curcumin analogues (UCAs) have been achieved. Tyrosinase inhibitory activities were found for most of the prepared synthetic UCAs. Among them, compounds containing 4-hydroxyl-substituted phenolic rings with $C$-2/C-4- or $C$-3/C-4-dihydroxyl-substituted diphenolic rings were more active $\left(\mathrm{IC}_{50}=1.74 \sim 16.74 \mu \mathrm{M}\right)$ than 4-butylresorcinol and kojic acid, which suggested that the 4-hydroxyl groups in UCAs play a crucial role in tyrosinase inhibitory activities. The inhibition kinetics analyzed by Lineweaver-Burk plots revealed compounds $\mathbf{3 c}$ and $\mathbf{3 i}$ containing catecholic rings were mixed-competitive inhibitors, whereas compounds $\mathbf{3 d}$ and 3j containing resorcinolic rings were competitive inhibitors. The preliminary evaluation results of acute toxicity showed the representative $\mathbf{3} \mathbf{d}$ and $\mathbf{3} \mathbf{j}$ were non-toxic in mice dosed at $1,200 \mathrm{mg} / \mathrm{kg}$. This research suggests that, with the advantage of being readily prepared small molecules, polyphenolic UCAs have the potential to develop into pharmacological inhibitors of tyrosinase.
\end{abstract}

Keywords: unsymmetrical curcumin analogues; synthesis; tyrosinase inhibitors; biological evaluation; inhibition kinetics 


\section{Introduction}

Generally, human skin color is determined by the type and the amount of melanin, a natural pigment produced by melanocytes, which plays a crucial role against skin photocarcinogenesis. However, abnormal melanin pigmentation can cause dermatological disorders such as lentigo, age spots, melasma, ephelide and senile lentigines [1,2]. Tyrosinase [EC1.14.18.1] is a key enzyme of the rate-limiting step for the biosynthesis pathway of melanin pigment. Tue to their potent activities tyrosinase inhibitors have been of particular interest both skin medications [3-5] and cosmetics [6,7] to prevent hyperpigmentation. Recently, various naturally occurring or synthetically produced tyrosinase inhibitors have been reported [2,8-12]. However, most of them suffered from limitations such as low activity, high toxicity and insufficient penetrative ability [13,14].

Curcumin, an antioxidant polyphenol from the rhizome of Curcuma longa Linn, is a major ingredient of turmeric, and it has been used for the therapy of inflammatory and infectious diseases in ayurvedic medicine [15]. Many studies showed that curcumin had cancer preventive [16], antiinflammatory [17], antioxidative [18] and antiviral activities [19]. In addition, the safety of curcumin is evident by its consumption for centuries at levels up to $100 \mathrm{mg}$ /day by people in some countries [20]. These beneficial properties have attracted numerous efforts for the development of curcumin as a safe therapeutic agent. Curcumin has been approved as a natural yellow color additive and antioxidant in cosmetics for many years [21]. Recently, curcumin was demonstrated to be beneficial to treat some skin diseases [22,23], and one of its derivatives, tetrahydrocurcumin, was recommended to be used in cosmetics as a lighting agent [24]. Furthermore, Lee et al recently reported that some curcumin analogues exhibited inhibitory activity against tyrosinase [25]. These reports attracted our interest to further study the inhibitory effect of curcumin analogues on tyrosinase.

In this study, considering polyphenolic compounds exhibited potent inhibitory activity against tyrosinase, and their 4-hydroxyl groups played a crucial role in some tyrosinase inhibitors [26-28], a series of unsymmetrical curcumin analogues (UCAs) bearing 4-hydroxyl groups were synthesized (Scheme 1) and their inhibitory activities against tyrosinase were evaluated. In addition, the inhibition mechanism and acute toxicity of several potent UCAs were also investigated in order to achieve our aims of developing novel tyrosinase inhibitors with potent activities and lower side effects.

\section{Results and Discussion}

\subsection{Chemistry}

The syntheses of polyphenolic UCAs (compounds $\mathbf{3 a}-\mathbf{k}$ and $\mathbf{4 a}-\mathbf{i}$, Scheme 1) were easily completed by a facile two-step sequence without the need for hydroxyl group protection. This began with an aldol condensation of an aromatic aldehyde (4-hydroxybenzaldehyde or 4-hydroxy-3-methoxy-benzaldehyde) with excess acetone or cyclopentanone under basic conditions (aq. $\mathrm{NaOH}$ ) to afford a conjugated enone $\mathbf{1}$ or $\mathbf{2}$. Finally, one more aldol condensation of a different aromatic aldehyde (using various substituted benzaldehyde derivatives) with the corresponding intermediate $\mathbf{1}$ or $\mathbf{2}$ under acidic conditions (catalytic amount of conc. $\mathrm{HCl}$ ) gave the desired UCAs $\mathbf{3}$ or $\mathbf{4}$ in $50 \sim 75 \%$ yield over two steps. Thus, twenty polyphenolic UCAs containing 4-hydroxyl groups on ring A were prepared. Among them, hydroxyl groups are only present at the $C-4$ position on ring A, while there are $C-4$ 
(e.g., 3c, 3i and 4f). Other substituents on ring A or B also influenced the inhibitory activities of UCAs. For instance, when the hydroxyl group at $C-3$ on ring $\mathrm{B}$ of $\mathbf{3 c}\left(\mathrm{IC}_{50}=6.78 \mu \mathrm{M}\right)$ was switched to a methoxyl group, the activity of the corresponding $\mathbf{3 b}\left(\mathrm{IC}_{50}=56.64 \mu \mathrm{M}\right)$ decreased ( $>8$-fold difference). When a bromine substituent was introduced into ring B, none of the bromides $(\mathbf{3 h}, \mathbf{3 g}$ and 4e) showed activities $(>170 \mu \mathrm{M})$. The bulky tert-butyl substituent on ring B also decreased the activity (3f, $\mathrm{IC}_{50}=168.36 \mu \mathrm{M}$ ). Interestingly, introduction of an additional methoxyl group into ring $\mathrm{B}$ of $\mathbf{3 b}$ led to a much more active compound $3 \mathbf{e}\left(\mathrm{IC}_{50}=9.66 \mu \mathrm{M}\right)$.

Figure 1. Effect of compound $\mathbf{3 d}$ on the diphenolase activity against mushroom tyrosinase for the catalysis of L-DOPA at $25^{\circ} \mathrm{C}$.

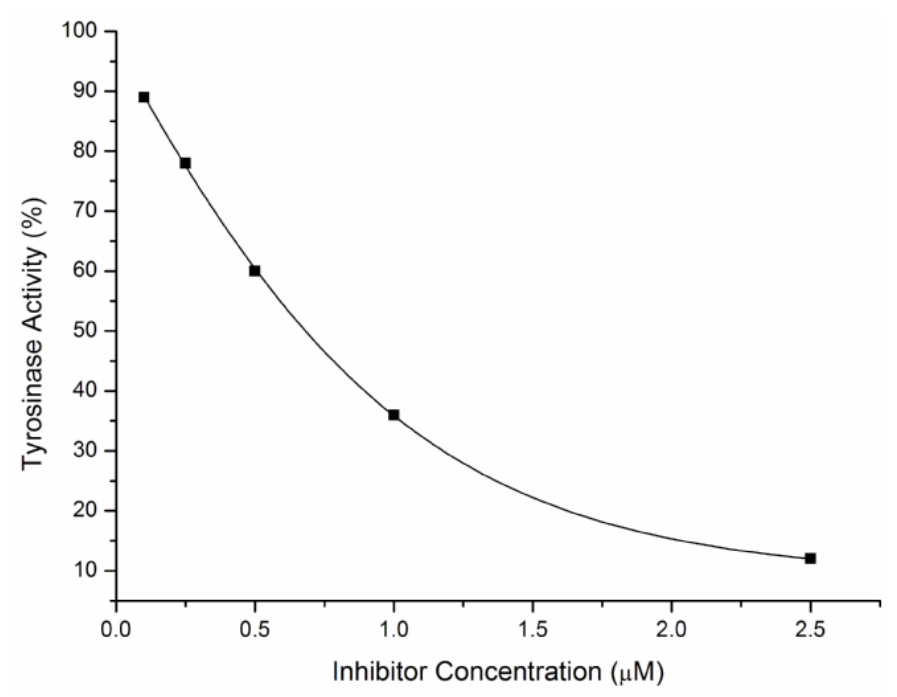

Table 1. Inhibitory effects of UCAs against mushroom tyrosinase.

\begin{tabular}{cccc}
\hline Compound & IC $_{\mathbf{5 0}}(\boldsymbol{\mu} \mathbf{M})$ & Compound & IC $_{\mathbf{5 0}}(\boldsymbol{\mu M})$ \\
\hline $\mathbf{3 a}$ & 189.42 & $\mathbf{4 a}$ & 182.86 \\
$\mathbf{3 b}$ & 56.64 & $\mathbf{4 b}$ & 46.24 \\
$\mathbf{3 c}$ & 7.78 & $\mathbf{4 c}$ & 4.64 \\
$\mathbf{3 d}$ & 1.74 & $\mathbf{4 d}$ & 7.20 \\
$\mathbf{3 e}$ & 9.66 & $\mathbf{4 e}$ & $>200$ \\
$\mathbf{3 f}$ & 168.36 & $\mathbf{4 f}$ & 16.34 \\
$\mathbf{3 g}$ & 173.48 & $\mathbf{4 g}$ & $>200$ \\
$\mathbf{3 h}$ & $>200$ & $\mathbf{4 h}$ & 86.92 \\
$\mathbf{3 i}$ & 16.74 & $\mathbf{4 i}$ & $>200$ \\
$\mathbf{3 j}$ & 2.78 & $4-B u t y l$ resorcinol & 11.27 \\
$\mathbf{3 k}$ & $>200$ & Kojic acid & 28.59 \\
\hline
\end{tabular}

\subsection{Kinetic Analysis of Selected Compounds on Mushroom Tyrosinase}

The inhibitory mechanism of selected compounds $\mathbf{3 c}, \mathbf{3 d}, \mathbf{3 i}$ and $\mathbf{3 j}$ against mushroom tyrosinase during the oxidation of L-DOPA was determined by the same methods. Double-reciprocal plots of the inhibition kinetics of selected compounds against tyrosinase are shown in Figure 2. Compounds $\mathbf{3 c}$ and 3i containing $o$-dihydroxyl groups on ring B were mixed-competitive inhibitors, as illustrated in Figure 
2 where increasing the concentration of $\mathbf{3 c}$ or $\mathbf{3 i}$ resulted in straight lines with different slopes and intercepts, while the lines were intersected in the second quadrant. However, $\mathbf{3 d}$ and $\mathbf{3 j}$ containing $m$-dihydroxyl groups on ring B were competitive inhibitors because the intersection of those straight lines was on the Y-axis.

Figure 2. Lineweaver-Burk plots for inhibition of compounds $\mathbf{3 c}, \mathbf{3 d}, \mathbf{3 i}$ and $\mathbf{3 j}$ against mushroom tyrosinase for the catalysis of L-DOPA. Concentrations of $\mathbf{3 c}, \mathbf{3 d}, \mathbf{3 i}$ and $\mathbf{3 j}$ for curves $1-3$ were $0.0 \mu \mathrm{M}, 4.06 \mu \mathrm{M}, 12.18 \mu \mathrm{M} ; 0.0 \mu \mathrm{M}, 4.06 \mu \mathrm{M}, 16.23 \mu \mathrm{M}$; $0.0 \mu \mathrm{M}, 14.8 \mu \mathrm{M}$, $59.2 \mu \mathrm{M} ; 0.0 \mu \mathrm{M}, 3.70 \mu \mathrm{M}, 11.09 \mu \mathrm{M}$, respectively.
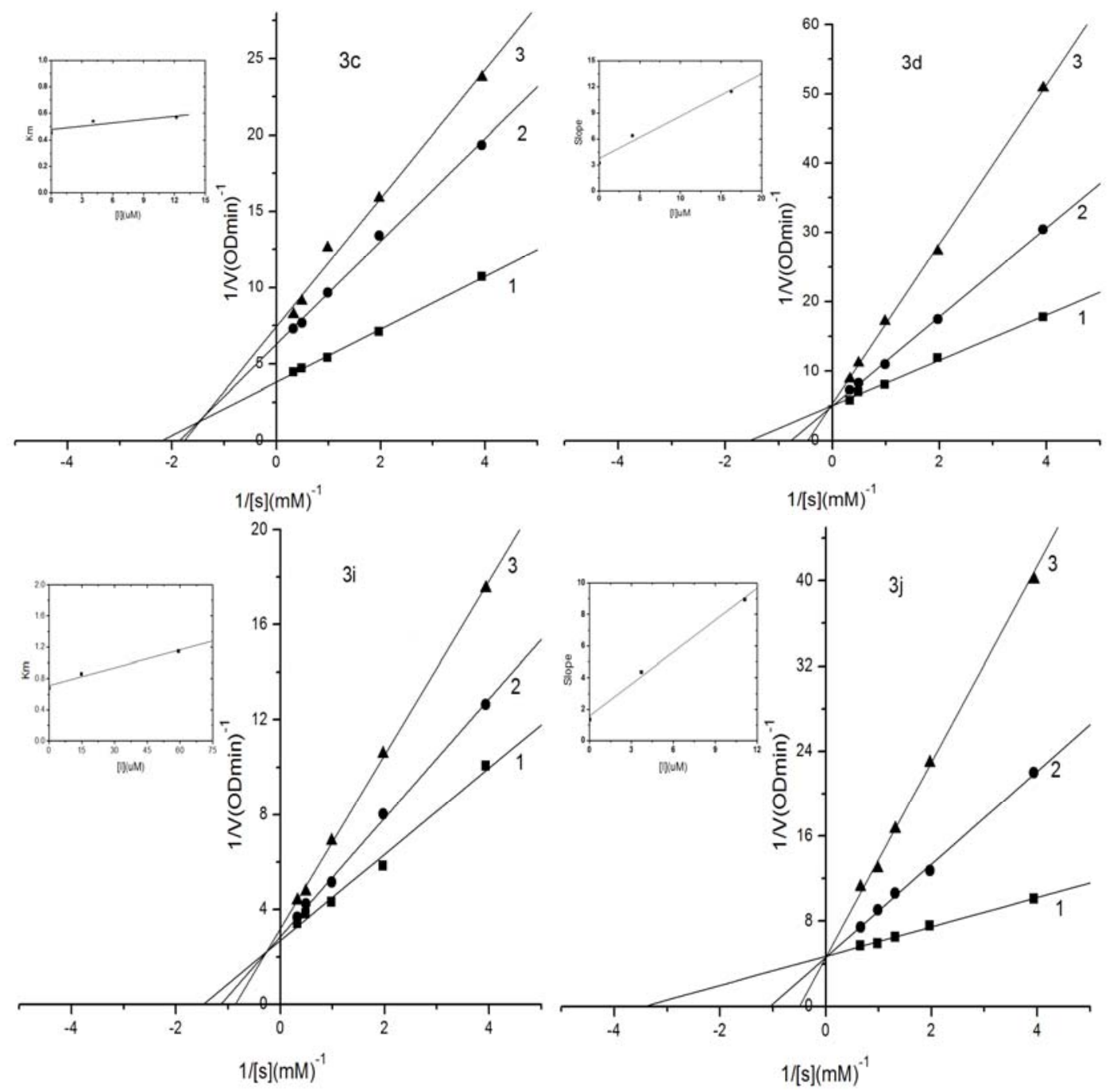

\subsection{Evaluation of Acute Toxicity in Mice}

3d and $\mathbf{3} \mathbf{j}$ were used as the representative compounds to evaluate the toxicity in mice. The experimental results demonstrated that daily dose of $1,200 \mathrm{mg} / \mathrm{kg}$ in mice led to no mortality for each mouse, and the observation of characteristics of mice in the control and treated groups, such as food intake, water drinking, body weight, general appearance, skin and fur, eyes and nose, respiration, urine, feces, locomotor, were not abnormal (Table 2). The autopsy results of all mice at the end of the experimental period (14 days) also revealed that no apparent changes were detected in any mice organs. These results indicated that $\mathbf{3 d}$ and $\mathbf{3 j}$ had no acute toxicity in mice at a dose of 1,200 $\mathrm{mg} / \mathrm{kg}$. 
Table 2. Observation of acute toxicity in mice for compound $\mathbf{3 d}$.

\begin{tabular}{|c|c|c|c|c|c|c|}
\hline \multirow[t]{2}{*}{ Item } & \multicolumn{2}{|c|}{ Control } & \multicolumn{2}{|c|}{ Treated by $3 d$} & \multicolumn{2}{|c|}{ Treated by $3 \mathbf{j}$} \\
\hline & Male & Female & Male & Female & Male & Female \\
\hline Food Intake (g/day) & $5.1 \pm 0.3$ & $3.9 \pm 0.4$ & $5.1 \pm 0.5$ & $4.0 \pm 0.4$ & $5.2 \pm 0.4$ & $3.9 \pm 0.5$ \\
\hline Drinking Water (mL/day) & $5.5 \pm 0.7$ & $4.2 \pm 0.4$ & $5.6 \pm 0.6$ & $4.2 \pm 0.5$ & $5.4 \pm 0.5$ & $4.1 \pm 0.4$ \\
\hline Body Weight (g) & $31.3 \pm 2.5$ & $27.1 \pm 2.3$ & $30.9 \pm 2.6$ & $27.3 \pm 2.9$ & $30.1 \pm 2.8$ & $26.9 \pm 2.1$ \\
\hline General Appearance & $\sqrt{ }$ & $\sqrt{ }$ & $\sqrt{ }$ & $\sqrt{ }$ & $\sqrt{ }$ & $\sqrt{ }$ \\
\hline Skin and Fur & $\sqrt{ }$ & $\sqrt{ }$ & $\sqrt{ }$ & $\sqrt{ }$ & $\sqrt{ }$ & $\sqrt{ }$ \\
\hline Eyes, Nose & $\sqrt{ }$ & $\sqrt{ }$ & $\sqrt{ }$ & $\sqrt{ }$ & $\sqrt{ }$ & $\sqrt{ }$ \\
\hline Respiration & $\sqrt{ }$ & $\sqrt{ }$ & $\sqrt{ }$ & $\sqrt{ }$ & $\sqrt{ }$ & $\sqrt{ }$ \\
\hline Urine & $\Delta$ & $\Delta$ & $\Delta$ & $\Delta$ & $\Delta$ & $\Delta$ \\
\hline Feces & $\Delta$ & $\Delta$ & $\Delta$ & $\Delta$ & $\Delta$ & $\Delta$ \\
\hline Locomotor & $\sqrt{ }$ & $\sqrt{ }$ & $\sqrt{ }$ & $\sqrt{ }$ & $\sqrt{ }$ & $\sqrt{ }$ \\
\hline
\end{tabular}

Note: $\sqrt{ }$ stands for Normal, and $\Delta$ stands for No Discoloration. CD-1 mice ( 9 males and 9 females; $7 \sim 8$ weeks old) were divided into 6 equal groups (controls: male, female, treated by $3 \mathrm{~d}$ : male and female, treated by $3 \mathrm{j}$ : male and female). Mice had free access to distilled water and commercial standard diet. Food Intake and Drinking Water were measured daily and averaged statistically, and Body Weight was measured in the last day. General Appearance, Skin, Fur, Eyes, Nose, Respiration, Urine, Feces and Locomotor were observed daily.

\subsection{Molecular Docking Study}

Recently, the crystallographic structure of tyrosinase has been revealed. The three-dimensional structure of tyrosinase enables us to gain a better understanding of the tyrosinase inhibition mechanism. Considering compounds $\mathbf{3 c} / \mathbf{3 i}$ and $\mathbf{3 d} / \mathbf{3} \mathbf{j}$ exhibited different inhibition mechanisms, we selected the representative compounds $\mathbf{3 c}$ and $\mathbf{3 d}$ to study the interaction mode by docking.

Although the structure of mushroom tyrosinase has not been determined, according to the reported crystallographic data, there is a high homology for the active center of most tyrosinases of different origin. In Figure 3, it was found that both $\mathbf{3 c}$ and $\mathbf{3 d}$ formed $\pi-\pi$ stacks between ring B of the inhibitors and HIS194 of tyrosinase, and there were hydrogen bonds between the 4-position phenolic groups of 3c/3d and the TRP184 residue of the active site of tyrosinase. This result further confirmed that hydroxyl groups in the 4-position of UCAs play a crucial role in the tyrosinase inhibitory activity.

The 4'-OH of 3c formed two hydrogen bonds with SER206 and the 3'-OH of 3c formed two hydrogen bonds with hydration water molecules of the binuclear copper ions of tyrosinase, while the distance between 3'-OH and copper ions CU502 was $2.90 \AA$ A (Figure 3, left). The 4'-OH of 3d formed a hydrogen bond with SER206 and another hydrogen bond with one hydration water molecule of the binuclear copper ions, while the distance between the 4'-OH and copper ion CU501 was $3.90 \AA$ (Figure 3, right), which is much weaker than that of 3c. Furthermore, the 2'-OH of 3d formed another hydrogen bond with ASN191. 
Figure 3. The proposed binding modes of 3c (left picture) and 3d (right picture) in the active site of tyrosinase (PDB access code 2ZWE). The inhibitor molecules are colored in yellow for carbon atoms. The dashed lines show hydrogen-bonding, the real lines show the distance of metal-coordination interactions. The docking models are generated using Surflex-Dock.
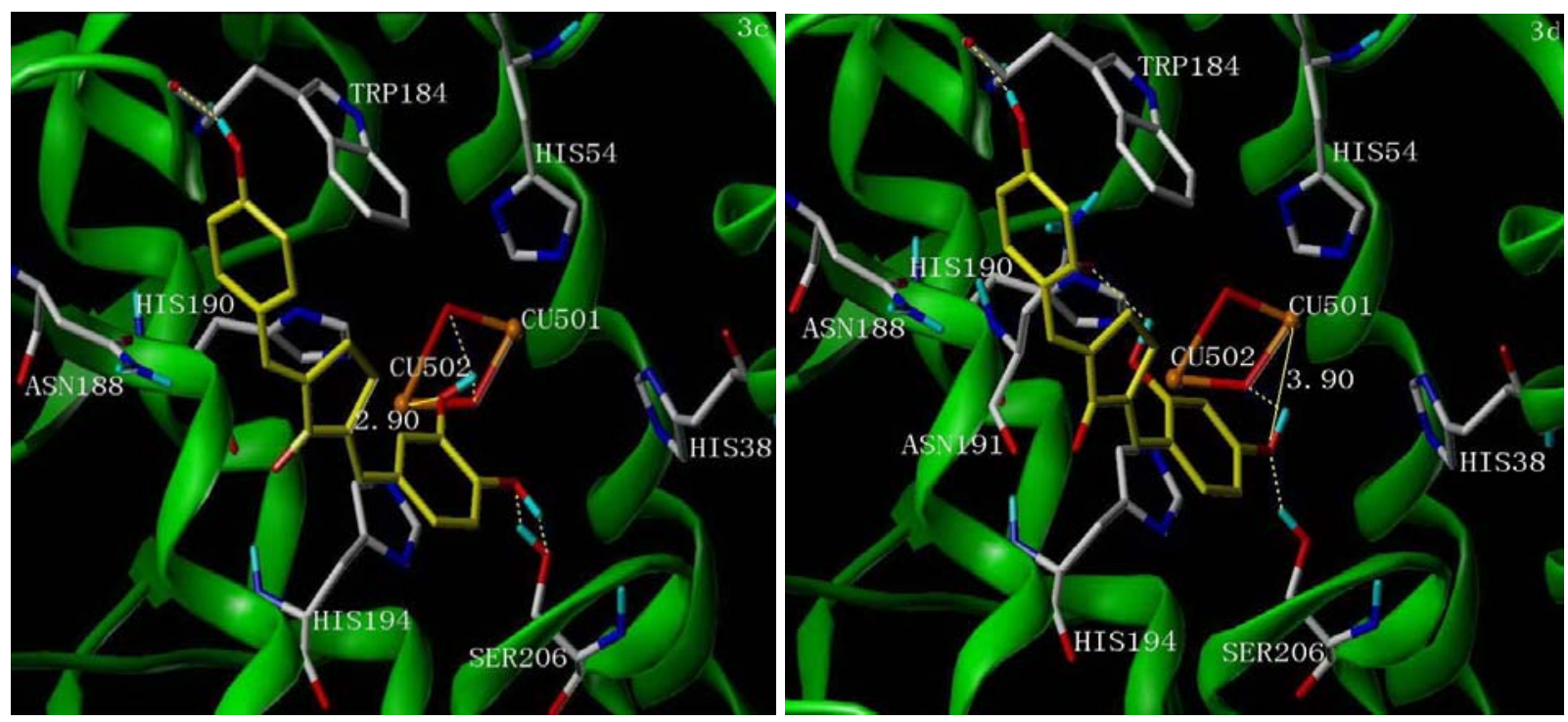

\section{Experimental}

\subsection{Reagents and General Procedures}

Melting points were determined on a Yanagimoto micro-melting apparatus and were uncorrected. The ${ }^{1} \mathrm{H}-\mathrm{NMR}$ spectra (300 MHz) were measured on a Varian Gemini-2000 spectrometer using DMSO- $d_{6}$ as solvent and TMS as an internal standard. Chemical shifts were expressed in ppm units. Multiplicities were recorded as s (singlet), brs (broad singlet), d (doublet), t (triplet), q (quartet), $\mathrm{m}$ (multiplet). Mass spectra were obtained on a LC-MS-2010A spectrometer with ESI. Elemental analyses were performed on a Perkin Elmer 240C instrument. Thin-layer chromatography (TLC) was performed on Merck silica gel plates (DC-60 F254).

\subsection{Synthesis}

The appropriate substituted benzaldehyde $(0.01 \mathrm{~mol})$ was dissolved in a mixture of aqueous $\mathrm{NaOH}$ [8\% (w/w), $16 \mathrm{~mL}]$ and ethanol $(10 \mathrm{~mL})$. The solution was then added dropwise to a stirred solution of acetone or cyclopentanone $(0.03 \mathrm{~mol})$ in aqueous $\mathrm{NaOH}[8 \%(\mathrm{w} / \mathrm{w}), 4 \mathrm{~mL}]$ at room temperature. The mixture was stirred at room temperature for $24 \mathrm{~h}$. The precipitation was collected by filtration, and the solid was then dissolved in a mixture solution of water and ethanol. The solution was neutralized by $10 \% \mathrm{HCl}$ in an ice-water bath to produce light yellow solid. The crude product was then filtered and recrystallized in ethanol to give the corresponding intermediate $\mathbf{1}$ or $\mathbf{2}$.

The intermediate 1 or $2(0.005 \mathrm{~mol})$ and the appropriate substituted benzaldehyde $(0.005 \mathrm{~mol})$ were dissolved in a small amount of ethanol or THF solvent. Concentrated $\mathrm{HCl}(0.1 \mathrm{~mL})$ was added and the mixture was stirred at room temperature for $0.5 \mathrm{~h}$. The reactant was then placed in a sealed container 
for 2 3 days and tracked by TLC until the reaction completed. The precipitation was collected by filtration, washed by cool ethanol and distilled water and recrystallized in an appropriate solvent or purified using silica gel flash chromatography to give UCAs 3 or 4.

2-(4-Hydroxybenzylidene)-5-(4-methoxybenzylidene) cyclopentanone (3a). Yield = 75\%; m.p.: 241-243 ${ }^{\circ} \mathrm{C}$; ${ }^{1} \mathrm{H}-\mathrm{NMR} \delta=10.02(\mathrm{br}, 1 \mathrm{H}), 7.63(\mathrm{~d}, J=8.7 \mathrm{~Hz}, 2 \mathrm{H}), 7.52(\mathrm{~d}, J=8.7 \mathrm{~Hz}, 2 \mathrm{H}), 7.34(\mathrm{~d}, J=6.6 \mathrm{~Hz}$, 2H), $7.03(\mathrm{~d}, J=8.7 \mathrm{~Hz}, 2 \mathrm{H}), 6.86(\mathrm{~d}, J=8.7 \mathrm{~Hz}, 2 \mathrm{H}), 3.90(\mathrm{~s}, 3 \mathrm{H}), 3.03$ (s, 4H); ESI-MS: $m / z=304.9$ $\left(\mathrm{M}^{+}-\mathrm{H}\right)$; Anal. Calc. for $\mathrm{C}_{20} \mathrm{H}_{18} \mathrm{O}_{3}$ : C, 78.41; H, 5.92. Found: C, 78.36; H, 5.95.

2-(4-Hydroxy-3-methoxybenzylidene)-5-(4-hydroxybenzylidene)cyclopentanone (3b). Yield = 72\%; m.p.: $278-280{ }^{\circ} \mathrm{C} ;{ }^{1} \mathrm{H}-\mathrm{NMR} \delta=10.01$ (brs, $\left.1 \mathrm{H}\right), 9.62$ (brs, $\left.1 \mathrm{H}\right), 7.52$ (d, $\left.J=8.7 \mathrm{~Hz}, 2 \mathrm{H}\right), 7.33-7.32$ $(\mathrm{m}, 2 \mathrm{H}), 7.22(\mathrm{~s}, 1 \mathrm{H}), 7.16-7.13(\mathrm{~m}, 1 \mathrm{H}), 6.86(\mathrm{dd}, J=8.4,2.7 \mathrm{~Hz}, 3 \mathrm{H}), 3.82(\mathrm{~s}, 3 \mathrm{H}), 3.03(\mathrm{~s}, 4 \mathrm{H})$; ESI-MS: $m / z=320.8\left(\mathrm{M}^{+}-\mathrm{H}\right)$; Anal. Calc. for $\mathrm{C}_{20} \mathrm{H}_{18} \mathrm{O}_{4}$ : C, 74.52; H, 5.63. Found: C, 74.49; H, 5.65.

2-(3,4-Dihydroxybenzylidene)-5-(4-hydroxybenzylidene)cyclopentanone $(\mathbf{3 c})$. Yield $=65 \%$; m.p.: $>300{ }^{\circ} \mathrm{C}$; ${ }^{1} \mathrm{H}-\mathrm{NMR} \delta=10.00(\mathrm{br}, 1 \mathrm{H}), 9.53$ (brs, $\left.1 \mathrm{H}\right), 9.19$ (brs, $\left.1 \mathrm{H}\right), 7.51(\mathrm{~d}, J=8.7 \mathrm{~Hz}, 2 \mathrm{H}), 7.30$ (s, 1H), 7.23 (s, 1H), $7.09(\mathrm{~d}, J=1.5 \mathrm{~Hz}, 1 \mathrm{H}), 7.00-6.97(\mathrm{~m}, 1 \mathrm{H}), 6.87(\mathrm{~s}, 1 \mathrm{H}), 6.84-6.80(\mathrm{~m}, 2 \mathrm{H}), 3.00(\mathrm{~s}, 4 \mathrm{H})$; ESI-MS: $m / z=306.8\left(\mathrm{M}^{+}-\mathrm{H}\right)$; Anal. Calc. for $\mathrm{C}_{19} \mathrm{H}_{16} \mathrm{O}_{4}$ : C, 74.01; H, 5.23. Found: C, 73.97; H, 5.24.

2-(2,4-Dihydroxybenzylidene)-5-(4-hydroxybenzylidene)cyclopentanone (3d). Yield = 68\%; m.p.: 241-243 ${ }^{\circ} \mathrm{C}$; ${ }^{1} \mathrm{H}-\mathrm{NMR} \delta=10.01$ (brs, $\left.1 \mathrm{H}\right), 9.97$ (brs, $\left.1 \mathrm{H}\right), 9.84$ (brs, $\left.1 \mathrm{H}\right), 7.73$ (s, 1H), 7.50 (d, J=8.7 Hz, 2H), $7.39(\mathrm{~d}, J=8.7 \mathrm{~Hz}, 1 \mathrm{H}), 7.28(\mathrm{~d}, J=2.1 \mathrm{~Hz}, 1 \mathrm{H}), 6.85(\mathrm{~d}, J=8.4 \mathrm{~Hz}, 2 \mathrm{H}), 6.42-6.30(\mathrm{~m}, 2 \mathrm{H})$, $3.00(\mathrm{~s}, 4 \mathrm{H})$; ESI-MS: $m / z=306.9\left(\mathrm{M}^{+}-\mathrm{H}\right)$; Anal. Calc. for $\mathrm{C}_{19} \mathrm{H}_{16} \mathrm{O}_{4}$ : C, 74.01; H, 5.23. Found: C, $73.95 ; \mathrm{H}, 5.25$.

2-(4-Hydroxy-3,5-dimethoxybenzylidene)-5-(4-hydroxybenzylidene)cyclopentanone (3e). Yield = 71\%; m.p.: 259-261 ${ }^{\circ} \mathrm{C} ;{ }^{1} \mathrm{H}-\mathrm{NMR} \delta=10.02$ (brs, 1H), 9.02 (brs, 1H), 7.52 (d, $\left.J=8.7 \mathrm{~Hz}, 2 \mathrm{H}\right), 7.33$ (m, 2H), $6.96(\mathrm{~s}, 2 \mathrm{H}), 6.86(\mathrm{~d}, J=8.7 \mathrm{~Hz}, 2 \mathrm{H}), 3.82(\mathrm{~s}, 6 \mathrm{H}), 3.08-3.03(\mathrm{~m}, 4 \mathrm{H})$; ESI-MS: $m / z=350.9\left(\mathrm{M}^{+}-\mathrm{H}\right)$; Anal. Calc. for $\mathrm{C}_{21} \mathrm{H}_{20} \mathrm{O}_{5}$ : C, 71.58; H, 5.72. Found: C, 71.50; H, 5.74.

2-(3,5-Di-tert-butyl-4-hydroxybenzylidene)-5-(4-hydroxybenzylidene)cyclopentanone (3f). Yield = 75\%; m.p.: $233-235{ }^{\circ} \mathrm{C} ;{ }^{1} \mathrm{H}-\mathrm{NMR} \delta=10.01(\mathrm{brs}, 1 \mathrm{H}), 7.66(\mathrm{~s}, 1 \mathrm{H}), 7.51(\mathrm{~d}, J=8.7 \mathrm{~Hz}, 1 \mathrm{H}), 7.42(\mathrm{~s}, 2 \mathrm{H})$, $7.33(\mathrm{~d}, J=8.7 \mathrm{~Hz}, 2 \mathrm{H}), 6.85(\mathrm{~d}, J=8.7 \mathrm{~Hz}, 2 \mathrm{H}), 3.03(\mathrm{~s}, 4 \mathrm{H}), 1.42$ (s, 18H); ESI-MS: $m / z=403.0$ $\left(\mathrm{M}^{+}-\mathrm{H}\right)$; Anal. Calc. for $\mathrm{C}_{27} \mathrm{H}_{32} \mathrm{O}_{3}$ : C, 80.16; H, 7.97. Found: C, 80.12; H, 8.00.

2-(3-Bromo-4-hydroxy-5-methoxybenzylidene)-5-(4-hydroxybenzylidene)cyclopentanone (3g). Yield = 75\%; m.p.: 241-243 ${ }^{\circ} \mathrm{C} ;{ }^{1} \mathrm{H}-\mathrm{NMR} \delta=10.10$ (brs, $\left.1 \mathrm{H}\right), 10.07$ (brs, $\left.1 \mathrm{H}\right), 7.52$ (d, $\left.J=8.7 \mathrm{~Hz}, 2 \mathrm{H}\right), 7.41$ $(\mathrm{s}, 1 \mathrm{H}), 7.34(\mathrm{~s}, 1 \mathrm{H}), 7.30(\mathrm{~s}, 1 \mathrm{H}), 7.27(\mathrm{~s}, 1 \mathrm{H}), 6.86(\mathrm{~d}, J=8.7 \mathrm{~Hz}, 2 \mathrm{H}), 3.88(\mathrm{~s}, 3 \mathrm{H}), 3.04(\mathrm{~s}, 4 \mathrm{H})$; ESI-MS: $m / z=399.0\left(\mathrm{M}^{+}-\mathrm{H}\right)$; Anal. Calc. for $\mathrm{C}_{20} \mathrm{H}_{17} \mathrm{BrO}_{4}$ : C, 59.87; H, 4.27. Found: C, 59.80; H, 4.29.

2-(3,5-Dibromo-4-hydroxybenzylidene)-5-(4-hydroxybenzylidene)cyclopentanone (3h). Yield = 69\%; m.p.: 286-288 ${ }^{\circ} \mathrm{C} ;{ }^{1} \mathrm{H}-\mathrm{NMR} \delta=9.75$ (brs, $\left.1 \mathrm{H}\right), 8.04$ (brs, $\left.1 \mathrm{H}\right), 7.83$ (s, 2H), 7.53 (d, $\left.J=8.7 \mathrm{~Hz}, 2 \mathrm{H}\right)$, $7.35(\mathrm{~s}, 1 \mathrm{H}), 7.26(\mathrm{~d}, J=1.2 \mathrm{~Hz}), 6.86(\mathrm{~d}, J=8.7 \mathrm{~Hz}, 2 \mathrm{H}), 3.02(\mathrm{~s}, 4 \mathrm{H})$; ESI-MS: $m / z=448.7\left(\mathrm{M}^{+}-\mathrm{H}\right)$; Anal. Calc. for $\mathrm{C}_{19} \mathrm{H}_{14} \mathrm{Br}_{2} \mathrm{O}_{3}$ : C, 50.70; H, 3.13. Found: C, 50.64; H, 3.14. 
2-(3,4-Dihydroxybenzylidene)-5-(4-hydroxy-3-methoxybenzylidene) cyclopentanone (3i). Yield = 60\%; m.p.: $>300{ }^{\circ} \mathrm{C} ;{ }^{1} \mathrm{H}-\mathrm{NMR} \delta=9.62$ (brs, $\left.1 \mathrm{H}\right), 9.54$ (brs, 1H), 9.19 (br, 1H), 7.32 (s, 1H), 7.23 (s, 2H), $7.14(\mathrm{~d}, J=8.7 \mathrm{~Hz}, 1 \mathrm{H}), 7.09(\mathrm{~s}, 1 \mathrm{H}), 6.98(\mathrm{~d}, J=8.7 \mathrm{~Hz}, 1 \mathrm{H}), 6.86(\mathrm{~d}, J=8.1 \mathrm{~Hz}, 1 \mathrm{H}), 6.81(\mathrm{~d}$, $J=8.1 \mathrm{~Hz}, 1 \mathrm{H}), 3.82(\mathrm{~s}, 3 \mathrm{H}), 3.03(\mathrm{~s}, 4 \mathrm{H})$; ESI-MS: $m / z=336.9\left(\mathrm{M}^{+}-\mathrm{H}\right)$; Anal. Calc. for $\mathrm{C}_{20} \mathrm{H}_{18} \mathrm{O}_{5}$ : C, 70.99; H, 5.36. Found: C, 70.90; H, 5.38.

2-(2,4-Dihydroxybenzylidene)-5-(4-hydroxy-3-methoxybenzylidene)cyclopentanone (3j). Yield = 60\%; m.p.: $223-225{ }^{\circ} \mathrm{C} ;{ }^{1} \mathrm{H}-\mathrm{NMR} \delta=10.06$ (brs, $\left.1 \mathrm{H}\right), 9.88$ (brs, 1H), 9.62 (brs, 1H), $7.76(\mathrm{~s}, 1 \mathrm{H}), 7.42(\mathrm{~d}$, $J=8.7 \mathrm{~Hz}), 7.32(\mathrm{~s}, 1 \mathrm{H}), 7.23(\mathrm{~s}, 1 \mathrm{H}), 7.12(\mathrm{~s}, 1 \mathrm{H}), 7.16(\mathrm{~d}, J=8.7 \mathrm{~Hz}, 1 \mathrm{H}), 6.86(\mathrm{~m}, 2 \mathrm{H}), 3.82$ (s, $3 \mathrm{H}), 3.03(\mathrm{~s}, 4 \mathrm{H})$; ESI-MS: $m / z=336.9\left(\mathrm{M}^{+}-\mathrm{H}\right)$; Anal. Calc. for $\mathrm{C}_{20} \mathrm{H}_{18} \mathrm{O}_{5}$ : C, 70.99; H, 5.36. Found: C, 70.86; H, 5.38.

2-(4-Hydroxy-3,5-dimethoxybenzylidene)-5-(4-hydroxy-3-methoxybenzylidene)cyclopentanone (3k). Yield $=65 \%$; m.p.: $129-131{ }^{\circ} \mathrm{C} ;{ }^{1} \mathrm{H}-\mathrm{NMR} \delta=9.67$ (brs, $\left.1 \mathrm{H}\right), 9.06$ (brs, $\left.1 \mathrm{H}\right), 7.37$ (s, 2H), $7.26(\mathrm{~s}, 1 \mathrm{H})$, $7.17(\mathrm{dd}, J=8.4,1.6 \mathrm{~Hz}, 1 \mathrm{H}), 6.99(\mathrm{~s}, 2 \mathrm{H}), 6.89(\mathrm{~d}, J=8.4 \mathrm{~Hz}, 1 \mathrm{H}), 3.83(\mathrm{~s}, 6 \mathrm{H}), 3.11(\mathrm{~m}, 4 \mathrm{H})$; ESIMS: $m / z=380.9\left(\mathrm{M}^{+}-\mathrm{H}\right)$; Anal. Calc. for $\mathrm{C}_{22} \mathrm{H}_{22} \mathrm{O}_{6}$ : C, 69.10; H, 5.80. Found: C, 69.02; H, 5.83.

1-(4-Hydroxyphenyl)-5-(4-methoxyphenyl)penta-1,4-dien-3-one (4a). Yield = 55\%; m.p.: 114-116 ${ }^{\circ} \mathrm{C}$; ${ }^{1} \mathrm{H}-\mathrm{NMR} \delta=10.04$ (brs, 1H), 7.73-7.69 (m, 3H), 7.65-7.59 (m, 3H), 7.15 (d, J=15.0 Hz), 7.09 (d, $J=15.0 \mathrm{~Hz}, 1 \mathrm{H}), 7.04(\mathrm{~d}, J=8.7 \mathrm{~Hz}, 2 \mathrm{H}), 6.81(\mathrm{~d}, J=8.7 \mathrm{~Hz}, 2 \mathrm{H}), 3.80(\mathrm{~s}, 3 \mathrm{H})$; ESI-MS: $m / z=279.1$ $\left(\mathrm{M}^{+}-\mathrm{H}\right)$; Anal. Calc. for $\mathrm{C}_{18} \mathrm{H}_{16} \mathrm{O}_{3}$ : C, 77.12; H, 5.75. Found: C, 77.04; H, 5.80.

1-(4-Hydroxy-3-methoxyphenyl)-5-(4-hydroxyphenyl)penta-1,4-dien-3-one (4b). Yield = 52\%; m.p.: 98-101 ${ }^{\circ} \mathrm{C} ;{ }^{1} \mathrm{H}-\mathrm{NMR} \delta=10.01$ (brs, $\left.1 \mathrm{H}\right), 9.62$ (brs, 1H), 7. 65-7.60 (m, 4H), $7.34(\mathrm{~s}, 1 \mathrm{H}), 7.19-7.16$ $(\mathrm{m}, 1 \mathrm{H}), 7.12-7.07(\mathrm{~m}, 2 \mathrm{H}), 6.82-6.80(\mathrm{~m}, 3 \mathrm{H}), 3.83\left(\mathrm{~s}, 3 \mathrm{H}\right.$ ESI-MS: $m / z=295.0\left(\mathrm{M}^{+}-\mathrm{H}\right)$; Anal. Calc. for $\mathrm{C}_{18} \mathrm{H}_{16} \mathrm{O}_{4}$ : C, 72.96; H, 5.44. Found: C, 72.84; H, 5.47.

1-(3,4-Dihydroxyphenyl)-5-(4-hydroxyphenyl)penta-1,4-dien-3-one $(4 \mathrm{c})$. Yield $=55 \%$; m.p.: $181-183{ }^{\circ} \mathrm{C}$; ${ }^{1} \mathrm{H}-\mathrm{NMR} \delta=7.69(\mathrm{~d}, J=15.3 \mathrm{~Hz}, 1 \mathrm{H}), 7.65(\mathrm{~d}, J=15.3 \mathrm{~Hz}, 1 \mathrm{H}), 7.58(\mathrm{~d}, J=8.1 \mathrm{~Hz}, 2 \mathrm{H}), 7.15$ (s, 1H), 7.08-7.06 (m, 2H), $7.00(\mathrm{~d}, J=15.3 \mathrm{~Hz}, 1 \mathrm{H}), 6.82-6.80(\mathrm{~m}, 3 \mathrm{H})$; ESI-MS: $m / z=280.8\left(\mathrm{M}^{+}-\mathrm{H}\right)$; Anal. Calc. for $\mathrm{C}_{17} \mathrm{H}_{14} \mathrm{O}_{4}$ : C, 72.33; H, 5.00. Found: C, 72.23; H, 5.03.

1-(4-Hydroxy-3,5-dimethoxyphenyl)-5-(4-hydroxyphenyl)penta-1,4-dien-3-one (4d). Yield = 50\%; m.p.: 93-95 ${ }^{\circ} \mathrm{C} ;{ }^{1} \mathrm{H}-\mathrm{NMR} \delta=10.04$ (brs, 1H), 9.01 (brs, 1H), $7.62(\mathrm{~m}, 4 \mathrm{H}), 7.07(\mathrm{~m}, 4 \mathrm{H}), 6.82(\mathrm{~s}, 2 \mathrm{H})$, $3.83(\mathrm{~s}, 6 \mathrm{H})$; ESI-MS: $m / z=325.1\left(\mathrm{M}^{+}-\mathrm{H}\right)$; Anal. Calc. for $\mathrm{C}_{19} \mathrm{H}_{18} \mathrm{O}_{5}$ : C, 69.93; H, 5.56. Found: C, 69.86 ; H 5.59 .

1-(3,5-Dibromo-4-hydroxyphenyl)-5-(4-hydroxyphenyl)penta-1,4-dien-3-one (4e). Yield = 51\%; m.p.: 110-113 ${ }^{\circ} \mathrm{C}$; ${ }^{1} \mathrm{H}-\mathrm{NMR} \delta=10.46$ (brs, $\left.1 \mathrm{H}\right), 10.06$ (brs, $\left.1 \mathrm{H}\right), 8.00$ (s, 2H), 7.70 (d, J=16.5 Hz, 1H), $7.60(\mathrm{~m}, 2 \mathrm{H}), 7.55(\mathrm{~d}, J=16.5 \mathrm{~Hz}, 1 \mathrm{H}), 7.30(\mathrm{~d}, J=16.2 \mathrm{~Hz}, 1 \mathrm{H}), 7.01(\mathrm{~d}, J=16.2 \mathrm{~Hz}, 1 \mathrm{H}), 6.82(\mathrm{~m}$, 2H); ESI-MS: $m / z=422.9\left(\mathrm{M}^{+}-\mathrm{H}\right)$; Anal. Calc. for $\mathrm{C}_{17} \mathrm{H}_{12} \mathrm{Br}_{2} \mathrm{O}_{3}$ : C, 48.15; H, 2.85. Found: C, 48.06; H, 2.88 . 
1-(4-Hydroxy-3-methoxyphenyl)-5-(4-methoxyphenyl)penta-1,4-dien-3-one (4f). Yield = 58\%; m.p.: 105-107 ${ }^{\circ} \mathrm{C}$; ${ }^{1} \mathrm{H}-\mathrm{NMR} \delta=9.62(\mathrm{brs}, 1 \mathrm{H}), 7.58(\mathrm{~m}, 3 \mathrm{H}), 7.15(\mathrm{~m}, 5 \mathrm{H}), 6.72(\mathrm{~m}, 3 \mathrm{H}), 3.80(\mathrm{~s}, 6 \mathrm{H})$; ESI-MS: $m / z=309.2\left(\mathrm{M}^{+}-\mathrm{H}\right)$; Anal. Calc. for $\mathrm{C}_{19} \mathrm{H}_{18} \mathrm{O}_{4}$ : C, 73.53; H, 5.85. Found: C, 73.50; H, 5.88.

1-(3,4-Dihydroxyphenyl)-5-(4-hydroxy-3-methoxyphenyl)penta-1,4-dien-3-one (4g). Yield = 50\%; m.p.: 214-216 ${ }^{\circ} \mathrm{C} ;{ }^{1} \mathrm{H}-\mathrm{NMR} \delta=7.70(\mathrm{~m}, 4 \mathrm{H}), 7.15(\mathrm{~d}, 2 \mathrm{H}), 7.09(\mathrm{~m}, 2 \mathrm{H}), 6.98(\mathrm{~m}, 3 \mathrm{H}), 6.50(\mathrm{~s}, 1 \mathrm{H})$, 3.87 (s, 3H); ESI-MS: $m / z=310.8\left(\mathrm{M}^{+}-\mathrm{H}\right)$; Anal. Calc. for $\mathrm{C}_{18} \mathrm{H}_{16} \mathrm{O}_{5}$ : C, 69.22; H, 5.16. Found: C, 69.15; H, 5.19.

1-(4-Hydroxy-3,5-dimethoxyphenyl)-5-(4-hydroxy-3-methoxyphenyl)penta-1,4-dien-3-one (4h). Yield $=56 \%$; m.p.: $100-102{ }^{\circ} \mathrm{C} ;{ }^{1} \mathrm{H}-\mathrm{NMR} \delta=9.75(\mathrm{~s}, 1 \mathrm{H}), 9.65(\mathrm{~s}, 1 \mathrm{H}), 7.63(\mathrm{~m}, 2 \mathrm{H}), 7.17(\mathrm{~m}, 4 \mathrm{H}), 7.07$ (s, $2 \mathrm{H}), 3.83(\mathrm{~d}, 9 \mathrm{H})$; ESI-MS: $m / z=354.9\left(\mathrm{M}^{+}-\mathrm{H}\right)$; Anal. Calc. for $\mathrm{C}_{20} \mathrm{H}_{20} \mathrm{O}_{6}: \mathrm{C}, 67.41 ; \mathrm{H}, 5.66$. Found: C, 67.32; H, 5.69.

1-(3,4-Dihydroxyphenyl)-5-(4-methoxyphenyl)penta-1,4-dien-3-one (4i). Yield =62\%; m.p.: 206-208 ${ }^{\circ} \mathrm{C}$; ${ }^{1} \mathrm{H}-\mathrm{NMR} \delta=7.68(\mathrm{~d}, J=16.5 \mathrm{~Hz}, 2 \mathrm{H}), 7.58(\mathrm{~d}, J=8.1 \mathrm{~Hz}, 2 \mathrm{H}), 7.15(\mathrm{~s}, 1 \mathrm{H}), 7.08-7.06(\mathrm{~m}, 2 \mathrm{H})$, 7.01-6.99 (m, 1H), $6.80(\mathrm{~d}, J=8.1 \mathrm{~Hz}, 2 \mathrm{H}), 6.79-6.77(\mathrm{~m}, 1 \mathrm{H}), 3.87(\mathrm{~s}, 3 \mathrm{H})$; ESI-MS: $\mathrm{m} / \mathrm{z}=294.8$ $\left(\mathrm{M}^{+}-\mathrm{H}\right)$; Anal. Calc. for $\mathrm{C}_{18} \mathrm{H}_{16} \mathrm{O}_{4}$ : C, 72.96; H, 5.44. Found: C, 72.87; H, 5.46.

\subsection{Tyrosinase Assay}

The spectrophotometric assay for tyrosinase was performed according to the reported method [26] with slight modifications. Briefly, all the synthesized compounds were screened for the diphenolase inhibitory activity of tyrosinase using L-DOPA as the substrate. All compounds were dissolved in DMSO. The final concentration of the test solution was $2.0 \%$. Phosphate buffer $(\mathrm{pH}=6.8)$ was used to dilute the DMSO stock solution of test compounds. Thirty units of mushroom tyrosinase $(0.5 \mathrm{mg} / \mathrm{mL})$ were firstly pre-incubated with these compounds in $50 \mathrm{mM}$ phosphate buffer $(\mathrm{pH}=6.8)$ at $25{ }^{\circ} \mathrm{C}$ for $10 \mathrm{~min}$. L-DOPA $(0.5 \mathrm{mM})$ was then added to the reaction mixture and the enzyme reaction was monitored by measuring the change in absorbance at $475 \mathrm{~nm}$ of the formation of L-DOPA chrome for $1 \mathrm{~min}$. The measurement was performed for three times for each concentration and averaged before further calculations. $\mathrm{IC}_{50}$ values were determined by the interpolation of the dose-response curves. Kojic acid was used as the standard inhibitor for tyrosinase.

\subsection{Inhibition Kinetics}

The determination of inhibition kinetics was performed by this method: for example, for each of three different inhibitor concentrations of compound 3d $(0.0 \mu \mathrm{M}, 4.06 \mu \mathrm{M}$ and $16.23 \mu \mathrm{M}$, respectively), L-DOPA concentration was varied (5, 10, 15, 20 and $25 \mu \mathrm{L})$. Pre-incubation and measurement time was the same as the procedure in section 3.3. Maximal initial velocity was determined from initial linear portion of absorbance between 0 and $60 \mathrm{~s}$ after addition of mushroom tyrosinase. The inhibition type on the enzyme was assayed by Lineweaver-Burk plots, and the inhibition constant was determined by the second plots of the apparent $\mathrm{Km} / \mathrm{Vm}$ or $1 / \mathrm{Vm}$ versus the concentration of compound. The same procedure was executed for other compounds. 


\subsection{Acute Toxicity Assays in Mice}

The acute toxicity of compound 3d was examined according to the OECD 423 Guideline for Testing of Chemicals Acute Oral Toxicity - Acute Toxic Class Method procedures [29]. The test procedure was applied with an initial dose at $1,200 \mathrm{mg} / \mathrm{kg}$. Male and female CD-1 mice (six males and six females; 7 8 weeks old) were obtained from Center of Animal Test of the Sun Yat-sen University, housed in the University-approved animal facility in rooms maintained at $22 \pm 2{ }^{\circ} \mathrm{C}$ with $55 \%-60 \%$ humidity and $12 \mathrm{~h}$ photoperiod. After five-day adaptation to laboratory conditions, mice were divided into four equal groups (controls: male, female, and treated: male and female, three animals in each group, equal body mass) and given by gavage either a single dosage of 1,200 $\mathrm{mg} \mathbf{3 d}$ (dispersed in 3\% Tween 80 aqueous solution)/kg, and equal volumes of distilled water containing 3\% Tween 80 for control groups, using a suitably graduated syringe and a stainless steel intubation cannula. Mice had free access to distilled water and commercial standard diet. Animals were observed individually after dosing at least once during the first $30 \mathrm{~min}$, periodically during the first $24 \mathrm{~h}$, with special attention given during the first $4 \mathrm{~h}$, and daily thereafter, for a total of 14 days. All observations were systematically recorded with individual records being maintained for each animal. Individual body weight of animals was determined shortly before the test substance was administered and weekly thereafter. All the animals were sacrificed at the end of the observation period and subjected to a necropsy. The same procedure was performed for compound $\mathbf{3 j}$.

\subsection{Molecular Modeling}

To date, any attempt to determine experimental X-ray structure of human tyrosinase has failed. To overcome this problem, a homology model was made with the crystal structure of a bacterium tyrosinase taken from Streptomyces castaneoglobisporus as template (PDB accession code 2ZWE). The crystal structure of tyrosinase 2 ZWE was a complex with caddie protein ORF378 which binded in the active site of tyrosinase. ORF378 was removed [30]. For docking study, all water molecules were removed, and AMBER charges were assigned, orientations of side chain amides were corrected, and hydrogens were added and their positions were optimized by energy minimization using AMBER7 FF99 force field. Compounds 3c and 3d were selected and built using the Sybyl 8.0 (Tripos, Inc., St. Louis, MO, USA). After sketching the molecules, Gasteiger-Hückel partial charges were automatically assigned. Energy evaluations were made using the Tripos force-filed. Geometry optimizations were performed using 20 iterations of simplex followed by 500 steps of steepest descent protocol and then 2,000 steps of Powell algorithm's minimization. The molecular energies of all compounds always converged within the gradient displacement criterion of $0.001 \mathrm{kcal} / \AA^{2}$. Docking calculations were performed with Surflex-Dock on the Red Hat workstation. "Protomol" of SurflexDock was used to guide the molecular docking. The protomol was defined by setting the threshold value and the bloat value at 0.66 and $2 \AA$, respectively. The binding pocket of tyrosinase has already been defined with the residues surrounding the dicopper ions since they are implied to the recognition of the L-DOPA substrate [30]. The values of additional starting conformations per molecule and maximum number of poses per ligand were both expanded to 40 to increase the accuracy of binding 
mode. Other parameters were based on the software default setting. Then the ligands were docked into the active side of tyrosinase under the same condition.

\section{Conclusions}

In summary, we have synthesized various poliphenolic unsymmetrical curcumin analogues (UCAs) using aldol condensation reactions. UCAs containing 4-hydroxyl-substituted phenolic rings were found to be potent tyrosinase inhibitors (with $\mathrm{IC}_{50}$ values up to $1.74 \mu \mathrm{M}$ ). The acute toxicity evaluation of $\mathbf{3 d}$ and $3 \mathbf{j}$ showed they were non-toxic in mice dosed at $1,200 \mathrm{mg} / \mathrm{kg}$. This work discloses a rapid assembly of UCAs and in turn. the possibility for numerous types of tyrosinase inhibitor studies. Efforts along these lines are ongoing and will be reported in due course.

\section{Acknowledgments}

This work was financially supported by National Natural Science Foundation of China (21272043, 81272452), Science and Technology Planning Project of Guangdong Province (2011B090400573, 2012B091000170), Guangdong Natural Science Foundation (S2011010004967), and supported by the State Key Laboratory of Natural and Biomimetic Drugs (K20120204).

\section{References}

1. Fistarol, S.K.; Itin, P.H. Disorders of pigmentation. J. Dtsch. Dermatol. Ges. 2010, 8, 187-201.

2. Chang, T.S. An updated review of tyrosinase inhibitors. Int. J. Mol. Sci. 2009, 10, 2440-2475.

3. Khan, M.T. Novel tyrosinase inhibitors from natural resources-their computational studies. Curr. Med. Chem.2012, 19, 2262-2672.

4. Ye, Y.; Chou, G.X.; Mu, D.D.; Wang, H.; Chu, J.H.; Leung, A.K.M.; Fong, W.F.; Yu, Z.L. Screening of Chinese herbal medicines for antityrosinase activity in a cell free system and B16 cells. J. Ethnopharmacol. 2010, 129, 387-390.

5. Davis, E.C.; Callender, V.D. Postinflammatory hyperpigmentation: A review of the epidemiology, clinical features, and treatment options in skin of color. J. Clin. Aesthet. Dermatol. 2010, 3, 20-31.

6. Gillbro, J.M.; Olsson, M.J. The melanogenesis and mechanisms of skin-lightening agentsexisting and new approaches. Int. J. Cosmet. Sci. 2011, 33, 210-221.

7. Smit, N.; Vicanova, J.; Pavel, S. The hunt for natural skin whitening agents. Int. J. Mol. Sci. 2009, 10, 5326-5349.

8. Parvez, S.; Kang, M.; Chung, H.S.; Bae, H. Naturally occurring tyrosinase inhibitors: Mechanism and applications in skin health, cosmetics and agriculture industries. Phytother. Res. 2007, 21, 805-816.

9. Kim, Y.J.; Uyama, H. Tyrosinase inhibitors from natural and synthetic sources: Structure, inhibition mechanism and perspective for the future. Cell Mol. Life Sci. 2005, 62, 1707-1723.

10. Wang, H.-M.; Chen, C.-Y.; Chen, C.-Y.; Ho, M.-L.; Chou, Y.-T.; Chang, H.-C.; Lee, C.-H.; Wang, C.-Z.; Chu, I.-M. (-)-N-Formylanonaine from Michelia alba as a human tyrosinase inhibitor and antioxidant. Bioorg. Med. Chem. 2010, 18, 5241-5247. 
11. Wang, H.-M.; Chen, C.-Y.; Wen, Z.-H. Identifying melanogenesis inhibitors from Cinnamomum subavenium with in vitro and in vivo screening systems by targeting the human tyrosinase. Exp. Dermatol. 2011, 20, 242-248.

12. Huang, H.-C.; Chang, T.-Y.; Chang, L.-Z; Wang, H.-F.; Yih, K.-H.; Hsieh, W.-Y.; Chang, T.-M. Inhibition of melanogenesis versus antioxidant properties of essential oil extracted from leaves of Vitex negundo Linn and chemical composition analysis by GC-MS. Molecules 2012, 17, 3902-3916.

13. Burnett, C.L.; Bergfeld, W.F.; Belsito, D.V.; Hill, R.A.; Klaassen, C.D.; Liebler, D.C.; Marks, J.G.; Shank, R.C.; Slaga, T.J.; Snyder, P.W.; et al. Final report of the safety assessment of kojic acid as used in cosmetics. Int. J. Toxicol. 2010, 29, 244S-273S.

14. Draelos, Z.D. Skin lightening preparations and the hydroquinone controversy. Dermatol. Ther. 2007, 20, 308-313.

15. Lopresti, A.L.; Hood, S.D.; Drummond, P.D. Multiple antidepressant potential modes of action of curcumin: A review of its anti-inflammatory, monoaminergic, antioxidant, immune-modulating and neuroprotective effects. J. Psychopharmacol. 2012, 26, 1512-1524.

16. Goel, A.; Aggarwal, B.B. Curcumin, the golden spice from Indian saffron, is a chemosensitizer and radiosensitizer for tumors and chemoprotector and radioprotector for normal organs. Nutr. Cancer 2010, 62, 919-930.

17. Aggarwal, B.B. Targeting inflammation-induced obesity and metabolic diseases by curcumin and other nutraceuticals. Annu. Rev. Nutr. 2010, 30, 173-199.

18. Zhou, H.; Beevers, C.S.; Huang, S. The targets of curcumin. Curr. Drug Targets 2011, 12, 332-347.

19. Marathe, S.A.; Datey, A.A.; Chakravortty, D. Herbal cocktail as anti-infective: Promising therapeutic for the treatment of viral diseases. Recent Pat. Antiinfect. Drug Discov. 2012, 7, 123-132.

20. Chainani-Wu, N. Safety and anti-inflammatory activity of curcumin: A component of tumeric (Curcuma longa). J. Altern. Complement. Med. 2003, 9, 161-168.

21. Suhaimi, H.; Ahmad, F.B.; Friberg, S.E. Curcumin in a model skin lotion formulation. J. Pharm. Sci. 1995, 84, 376-380.

22. Thangapazham, R.L.; Sharad, S.; Maheshwari, R.K. Skin regenerative potentials of curcumin. Biofactors 2013, 39, 141-149.

23. Lima, C.F.; Pereira-Wilson, C.; Rattan, S.I. Curcumin induces heme oxygenase-1 in normal human skin fibroblasts through redox signaling: Relevance for anti-aging intervention. Mol. Nutr. Food Res. 2010, 55, 430-442.

24. Asawanonda, P.; Klahan, S.O. Tetrahydrocurcuminoid cream plus targeted narrowband UVB phototherapy for vitiligo: A preliminary randomized controlled study. Photomed. Laser Surg. 2010, 28, 679-684.

25. Lee, K.H.; Ab Aziz, F.H.; Syahida, A.; Abas, F.; Shaari, K.; Israf, D.A.; Lajis, N.H. Synthesis and biological evaluation of curcumin-like diarylpentanoid analogues for anti-inflammatory, antioxidant and anti-tyrosinase activities. Eur. J. Med. Chem. 2009, 44, 3195-3200.

26. Bao, K.; Dai, Y.; Zhu, Z.B.; Tu, F.J.; Zhang, W.G.; Yao, X.S. Design and synthesis of biphenyl derivatives as mushroom tyrosinase inhibitors. Bioorg. Med. Chem. 2010, 18, 6708-6714.

27. Yamazaki, Y.; Kawano, Y. Inhibitory effect of hydroxyindoles and their analogues on human melanoma tyrosinase. Z. Naturforsch. C 2010, 65, 49-54. 
28. Yi, W.; Cao, R.; Peng, W.; Wen, H.; Yan, Q.; Zhou, B.; Song, H. Synthesis and biological evaluation of novel 4-hydroxybenzaldehyde derivatives as tyrosinase inhibitors. Eur. J. Med. Chem. 2010, 45, 639-646.

29. OECD. Testing of Chemicals Acute Oral Toxicity-Acute Toxic Class Method. In OECD Guidelines for the Testing of Chemicals (E-book); OECD Publishing: Paris, France, 2001; pp. 1-14.

30. Matoba, Y.; Kumagai, T.; Yamamoto, A.; Yoshitsu, H.; Sugiyama, M. Crystallographic evidence that the dinuclear copper center of tyrosinase is flexible during catalysis. J. Biol. Chem. 2006, 281, 8981-8990.

Sample Availability: Samples of the compounds $\mathbf{3 a}-\mathbf{k}$ and $\mathbf{4 a - i}$ are available from the authors.

(C) 2013 by the authors; licensee MDPI, Basel, Switzerland. This article is an open access article distributed under the terms and conditions of the Creative Commons Attribution license (http://creativecommons.org/licenses/by/3.0/). 